\title{
Representaciones de democracia y ciudadanía en estudiantes universitarios de pedagogía del sur de Chile
}

Social representations of democracy and citizenship in southern Chile's undergraduate pedagogy students

\author{
Volumen 17, Número 2 \\ Mayo- Agosto \\ pp. 1-28
}

Este número se publica el $1^{\circ}$ de mayo de 2017

DOI: http://dx.doi.org/10.15517/aie.v17i1.28202

\author{
David Chávez Herting \\ Manuel Santiago Mieres Chacaltana
}

Revista indizada en REDALYC, $\underline{\text { SCIELO }}$

Revista distribuida en las bases de datos:

\section{LATINDEX, DOAJ, REDIB, IRESIE, CLASE, DIALNET, SHERPA/ROMEO, QUALIS-CAPES, MIAR}

Revista registrada en los directorios:

ULRICH'S $, \underline{R E D I E}, \underline{\text { RINACE}}, \underline{\text { OEI }}$ MAESTROTECA, PREAL, $\underline{\text { CLACSO }}$ 


\title{
Representaciones de democracia y ciudadanía en estudiantes universitarios de pedagogía del sur de Chile
}

Social representations of democracy and citizenship in southern Chile's undergraduate pedagogy students

\author{
David Chávez Herting ${ }^{1}$ \\ Manuel Santiago Mieres Chacaltana ${ }^{2}$
}

Resumen: El presente artículo presenta los resultados de una investigación cuyo objetivo fue determinar y describir las representaciones sobre democracia y ciudadanía de un conjunto de estudiantes universitarios de Pedagogía, con la finalidad de aportar antecedentes para el debate con respecto a la relevancia de incluir una estrategia clara de formación ciudadana en estas carreras. El estudio tiene un enfoque metodológico cualitativo. Se aplicó a 249 estudiantes pertenecientes a la Facultad de Educación de una universidad regional del sur de Chile. Se utilizó la técnica de redes semánticas naturales y el análisis se realizó tomando los valores arrojados por dichas redes, indagando sobre las representaciones de "democracia", "ciudadanía", "partidos políticos chilenos" y "democracia en Chile". Los resultados evidencian que los jóvenes representan la democracia como un adecuado sistema de organización social y política, y la ciudadanía como su forma de expresión válida. Sin embargo, al focalizar la atención en la realidad chilena, desacreditan la expresión que adquiere la democracia en el país, con representaciones negativas de los partidos políticos chilenos también. Se observa una clara ruptura entre los conceptos ideales de democracia y ciudadanía, y su aplicación concreta a la realidad chilena. Estos resultados permiten una primera aproximación a las conceptualizaciones políticas de los estudiantes con miras al diseño de una política de formación inicial docente que incluya la formación ciudadana.

Palabras clave: representaciones sociales, democracia, ciudadanía, estudiantes universitarios.

Abstract: This article presents the results of an investigation whose objective was to identify and describe the representations about democracy and citizenship of a group of undergraduate pedagogy's students, in order to provide a first background for the discussion on the relevance to include a clear strategy of citizenship education for these careers. The study has a qualitative methodological approach. It was applied to 249 students from the Faculty of Education at a regional university of southern Chile. The technique used was a Natural Semantic Network and analysis was made on the values thrown by such networks. We inquired about the representations of "democracy", "citizenship", "Chilean political parties" and "democracy in Chile". The results show that young people represent democracy as an adequate system of social and political organization and citizenship as its valid form of expression. However, by focusing attention on the Chilean reality, the expression of democracy in the country is discredited. They also represent negatively Chilean political parties. We observed a clear break between the ideals of democracy and citizenship concepts and their concrete application to the Chilean reality. These results allow a first approach to the political conceptualizations of students to design a policy of initial teacher training that includes civic education.

Keywords: social representations, democracy, citizenship, undergraduate students.

\footnotetext{
${ }^{1}$ Estudiante de doctorado en Psicología de la Comunicación y Cambio, Universidad Autónoma de Barcelona, Barcelona, España. Dirección electrónica: dchavez.herting@gmail.com
}

2 Académico adjunto de la Universidad Católica de Temuco, Chile. Profesor de estado en Historia, Geografía y Ciencias Sociales. Dirección electrónica: mieres@uct.cl

Artículo recibido: 21 de junio, 2016

Enviado a corrección: 20 de octubre, 2016

Aprobado: 23 de enero, 2017 


\section{Introducción}

Desde hace un par de décadas, la comunidad internacional ha venido desarrollando ingentes esfuerzos por promover la democracia, como lo demuestran los sucesivos informes del Programa de las Naciones Unidas para el Desarrollo (PNUD) del último tiempo. En efecto, hacia el 2002, 140 países se tipificaban como democráticos, aunque solo 82 de ellos disfrutaban de plena democracia (PNUD, 2002). En América Latina, de 18 países estudiados en 2004, todos desplegaban los requisitos fundamentales del régimen democrático; mientras que 25 años antes solo 3 de ellos cumplían con esa condición (PNUD, 2004). Sin embargo, estos avances han estado amenazados por las grandes expresiones de pobreza y desigualdad de la región. Varios estudios sugieren que las variables socioeconómicas inciden en la percepción y la calidad de la democracia (e.g., Redón, 2010; Taguenca y Lugo, 2011). Aun cuando en la última década se han experimentado notables mejoras en materia de desarrollo social, todavía resultan insuficientes (PNUD, 2011; 2014a).

Otro problema general de las democracias contemporáneas es el desfase entre las campañas electorales y la implementación de políticas verdaderamente públicas. Las campañas contribuyen a posicionar una agenda y una validación del sistema político a través de las elecciones. Sin embargo, con posterioridad, la ciudadanía tiene escasa o nula participación en la formulación e implementación de la agenda política (Dorantes, 2014). Frente a ello, los sectores excluidos tienden a alejarse de la política electoral, organizar protestas y movimientos sociales, y judicializar la política (Domingo, 2009).

En Chile, la ciudadanía también ha estado excluida de la construcción del orden social, acarreando la ausencia de legitimidad (Salazar y Pinto, 1999). En su historia reciente, el ejemplo más evidente y profundo es el periodo de la dictadura militar que significó, además, una fractura de la identidad nacional (Larraín, 2011) y la construcción de una plataforma ideológica enfocada en el nacionalismo y el disciplinamiento social, dificultando la recuperación de una atmósfera cultural democrática (Errázuriz, 2009). Aunque el país ha experimentado éxito económico, no ha logrado institucionalizar valores democráticos en la ciudadanía (Aparecido y Borba, 2011), y la democracia registra un apoyo moderado en relación con otros países de la región (Aravena, 2011). La prioridad dada al mercado por sobre las necesidades de la sociedad y la falta de mayor democratización del sistema político concentran el malestar ciudadano (De la Cuadra, 2013). El sistema de partidos políticos es evaluado como uno de los más estables de América Latina (Passarelli, 2011), pero desarraigado de la base social (Díaz y Sierra, 2012). Algunos autores destacan el 
proceso paradójico de recuperación de la democracia, en el que todas las decisiones fueron tomadas por la esfera política, sin la participación ciudadana que buena parte de la población esperaba (Flores-González y García-González, 2014).

Consecuencias evidentes de lo anterior son la pérdida de confianza en la política institucional y la gestación de mecanismos de resistencia alternativos como la protesta y los movimientos sociales (Cabalin, 2012; De la Cuadra, 2013; Jara, 2014), especialmente por parte de los jóvenes, quienes lograron instaurar un movimiento estudiantil de gran impacto en el país (Flores-González y García-González, 2014). Un estudio revela que los demócratas chilenos que no confían en los partidos son manifestantes activos, pero que tienden a evitar la política electoral (Carlin, 2011). Otro estudio desarrollado en 12 universidades de la capital señala que los jóvenes que se identifican políticamente tienden a ser tan críticos como los que no se identifican políticamente (González et al., 2005).

La juventud chilena también muestra señales de malestar frente a la política institucional y sus representantes (Flores-González y García-González, 2014; Instituto Nacional de La Juventud [INJUV], 2013), siendo un sector que ha sido sistemáticamente excluido y estereotipado, negando su diversidad y sus múltiples expresiones de participación (Adaros, Manzano, Mella y Montero, 2010). Este malestar no significa desinterés en la política. Los jóvenes parecen optar por expresiones alternativas de participación política, como la protesta, los movimientos sociales y la articulación colectiva a través de medios digitales (INJUV, 2013). En un estudio, los jóvenes califican la política chilena de rígida, corrupta, discriminadora, centralizada y lejana, aunque importante y necesaria (Cárdenas, Parra, Picón, Pineda y Rojas, 2007).

En Chile hay cierto consenso en que la enseñanza de la ciudadanía debe desarrollarse en el ámbito escolar (Comisión Formación ciudadana, 2004; INJUV, 2013). Sin embargo, el problema es consensuar los saberes que deben sostener proyectos de este tipo (González, 2014). En otras palabras, definir qué tipo de ciudadanía debe enseñarse (Candia y Gomes, 2013). Este problema es complejo porque la educación ciudadana es, en primer lugar, una educación en valores (Guichot, 2013; Martínez, 2011) que puede asumir variadas significaciones. Otra dificultad radica en el desmoronamiento de la base del orden social sustentado en la escuela y la familia (Centeno, 2008).

En general, el impacto de la escuela en materia de educación cívica ha sido lento e insuficiente. En las dos evaluaciones sobre el tema a las que se ha sometido Chile, en los años 1999 y 2009, no hubo progreso alguno en los resultados obtenidos (Flores-González y García-González, 2014) - resultados, por lo demás, muy por debajo del promedio 
internacional. Para algunos, esto se debe a formas de enseñanza y evaluación muy tradicionales (Agüero, Araya, Marín, Molina y Rojas, 2011). Otros, apuntan a las estructuras y prácticas de la institución educativa, que reproducen esquemas de exclusión, dominación y violencia provenientes del propio contexto societal (Fernández-Herrería y López-López, 2014; Redón, 2010; Reyes, Campos, Osandón y Muñoz, 2013). Finalmente, algunos sostienen que el problema radica en la formación inicial, que no permite que los docentes se apropien de contenidos ni estrategias adecuadas para educar en ciudadanía (Reyes et al., 2013).

En materia de propuestas, algunos postulan vincular la formación ciudadana con la actual cultura digital a fin de promover una "ciudadanía digital" difusora y defensora de los ideales democráticos (Gozálvez, 2011), o que los movimientos sociales sean vistos como un dispositivo de formación ciudadana (Delgado, 2011). Otros postulan un aprendizaje de la ciudadanía a través de acciones de servicio destinadas a distintos grupos de una comunidad (Puig, Gijón, Martín y Rubio, 2010).

En el ámbito de la educación superior una investigación realizada en Chile con estudiantes de pedagogía releva la labor de modelaje que desempeñan los profesores en sus procesos formativos, valorando la idoneidad pedagógica y la disposición hacia las buenas relaciones con sus alumnos (Cárcamo y Méndez, 2016). No obstante, la cultura académica suele enfatizar la instrucción y la investigación, en desmedro de otras actividades relacionadas con una misión más ético-moral (Bernardo, 2014; Macfarlane, 2007), como la formación ciudadana y que, por otra parte, no son explicitadas en los roles de los académicos (Lawrence, Ott y Bell, 2011).

Recientemente, el Congreso Nacional de Chile (2016) ha promulgado la Ley 20.911 la cual crea el plan de formación ciudadana para los establecimientos educacionales reconocidos por el Estado. Al respecto, el texto sostiene en su artículo único que:

(...) los establecimientos educacionales reconocidos por el Estado deberán incluir en los niveles de enseñanza parvularia, básica y media un Plan de Formación Ciudadana, que integre y complemente las definiciones curriculares nacionales en esta materia, que brinde a los estudiantes la preparación necesaria para asumir una vida responsable en una sociedad libre y de orientación hacia el mejoramiento integral de la persona humana, como fundamento del sistema democrático, la justicia social y el progreso. (p. 2) 
En razón de lo anterior, se refuerza la necesidad de construir propuestas que, desde el sistema escolar, contribuyan a fortalecer los procesos de formación ciudadana y su inclusión en la formación inicial docente. Para ello se requiere, entre otras cosas, indagar en las ideas que los futuros maestros y maestras tienen de la política y la ciudadanía.

\section{Marco teórico}

Un rasgo fundante de la democracia reside en la existencia de una comunidad política. En ella, los ciudadanos participan en la conducción política de la colectividad a la que pertenecen (Lizcano, 2012). La participación se ejerce en el espacio público, donde tienen lugar las discusiones sobre los asuntos del Estado (Taguenca, 2012). El cómo las personas se acercan y apropian de este espacio público se explica desde las condiciones institucionales objetivas creadas por el marco jurídico político. Pero también, a partir de una base asociativa más o menos espontánea que recoge problemas generados en el ámbito privado y lo transmite al espacio de opinión pública-política (Habermas, 1998). Cuando esta acción colectiva es capaz de fiscalizar y controlar a los cuadros que la representan en las estructuras gubernamentales, el sistema político adquiere un carácter de legitimidad (Habermas, 1998; Salazar y Pinto, 1999). Con ello la legitimidad aparece asociada a un poder conferido por la base comunitaria (Salazar, 2012). Si la expresión ciudadana es coartada por el derecho vigente y el Estado se construye con prescindencia de la participación civil, entonces, tarde o temprano, la ciudadanía tenderá a desacatar el orden legal e instalar su propia legitimidad (Habermas, 1998; Rosanvallon, 2007; Salazar 2009).

Desde la teoría, también es relevante destacar el principio de organización de la formación social del capitalismo liberal el cual descansa en la relación entre trabajo asalariado y capital (Habermas, 1975). Dicha situación constituye una base de desigualdad, pues "en el mercado de trabajo no se intercambian equivalentes" (Habermas, 1975, p. 43), deviniendo finalmente en "una producción socializada que se realiza en favor de intereses no generalizables" (Habermas, 1975, p. 65). En dicho marco de acción social se produce la resistencia (Janoschka, 2011; Rosanvallon, 2007).

En consecuencia, el actual contexto epocal demanda la formación de ciudadanos para un mundo global (Cortina, 1998; Re, 2014). El marco de la globalización neoliberal obliga a repensar la ciudadanía como una "categoría práctica que se expresa a través de sitios y actos específicos y que por tanto debe ser constantemente actualizada" (Sequera y 
Janoschka, 2012, p. 517). En su expresión meramente política la ciudadanía está siendo ampliamente superada (Garretón, 2003).

Martins (2000) distingue tres significaciones de la ciudadanía. La primera, como valor económico, enfatiza la dimensión económica por sobre las demás, reduciendo al ciudadano a un consumidor de los productos y servicios del Estado. La segunda es la significación gnoseológica, que hace referencia al ciudadano conocedor de sus derechos y obligaciones. No obstante, su posición frente a la realidad es acrítica, no cuestiona el orden establecido ni participa de su construcción. Por último, la ciudadanía puede ser concebida como valor ético y político. Desde este enfoque el ciudadano, conocedor de derechos y deberes, es además un participante activo de la vida pública en pro del cambio social para el beneficio de la colectividad. La dimensión ética de la ciudadanía también es enfatizada por autores como Cortina (2011) quien argumenta que el Estado y la esfera pública se nutren de marcos éticos mínimos y máximos.

El denominado debate liberal-comunitarista también ha servido de marco para posicionar puntos centrales referidos al concepto de ciudadanía. El liberalismo levanta una estructura conceptual sostenida en cuatro grandes ideas. En primer lugar, está la seguridad, considerada la base de la legitimidad de la organización política. Segundo, el derecho a la intimidad y su preferencia sobre el ámbito de lo público. En tercer término, la reivindicación de la libertad y la autonomía. Finalmente, el principio de neutralidad del gobierno frente a las distintas concepciones de vida buena y bien moral individual (Bárcena, 1997).

John Rawls (1995b), referente central del liberalismo político, sostiene la necesidad de edificar un sistema de justicia que sirva como estructura básica de la sociedad. En esta perspectiva, los ciudadanos se organizarían bajo dos principios esenciales. Según el primero, cada persona ha de poseer las libertades fundamentales que compatibilice con un esquema semejante de libertades para los demás. De acuerdo al segundo, las desigualdades económicas y sociales deben organizarse según un modelo que garantice a todos un acceso ventajoso a empleos y cargos (Rawls, 1995b). Bajo esta organización primordial los ciudadanos pueden edificar diversos proyectos de vida según sean sus concepciones de bien. Sin embargo, las ideas de bien admisibles deben respetar los límites impuestos por los mínimos de justicia antes señalados (Rawls, 1995a).

El enfoque comunitarista sostiene que al centrar el foco en el individuo, el liberalismo favorece la atomización y la disgregación social (Cerda, Egaña, Magendzo, Santa Cruz y Varas, 2004). El individuo prioriza su bien por sobre el de los demás. 
El comunitarismo, por su parte, enfatiza el sentido de pertenencia a la comunidad como base fundamental de una sociedad democrática. La pertenencia es un mecanismo generador de identidad y compromete al ciudadano con la comunidad, pues su identidad se construye en el marco del mundo social (Walzer, 1996). La materialización de dicho vínculo queda expresada a través de la participación política. La definición de virtudes, como cualidades necesarias, no se elabora individualmente, sino en el marco de una vida comunitaria contextualizada históricamente (Maclntyre, 2004).

En este contexto, toma fuerza la noción de "representaciones sociales", entendidas como "un sistema de valores, nociones y prácticas que proporciona a los individuos los medios para orientarse en el contexto social y material para dominarlo" (Moscovici, 1979, p. 24), expresándose por tanto a través de valores, opiniones, creencias, informaciones, imágenes, actitudes, categorías, esquemas, estereotipos o juicios (Hewstone, 1982, 1988; Moscovici, 2001). Estas representaciones se describen como proposiciones, reacciones y evaluaciones que se expresan en un conjunto de opiniones características de cada grupo social, permitiendo una aproximación a los significados compartidos (lbáñez, 1988). Implica, por lo tanto, una construcción simbólica del sentido que se les asigna a las instituciones y a los hechos, derivado de los diversos contextos sociales en los que los individuos se ubican. (Berger y Luckmann, 1991)

Para comprender el fenómeno de las representaciones sociales, conviene recordar que se han identificado por lo menos tres dimensiones, que en su interacción conforman lo que entendemos como representación social (Moscovici, 1979, 2001): actitud, información y campos de representación. Para efectos de esta investigación, se ha considerado que el campo de representación constituye el verdadero núcleo organizador y descriptivo de la representación social. De hecho, se evalúa el sentido social de las representaciones sociales con base en las categorías semánticas que lo forman. Estructuralmente, es posible imaginar el campo de representación como un sistema con un núcleo figurativo alrededor del cual se organiza elementos periféricos que permiten integrar nuevas informaciones o modificaciones (Condor y Antaki, 2000). Hoy es posible hablar de la relevancia de las representaciones sociales en la modulación, por cierto no determinística, de la construcción del conocimiento social (Castorina, 2008). No obstante, todo el funcionamiento político de los individuos en sus procesos de crecimiento vital depende en gran medida de estas construcciones mediadas por el entorno social. Es más, se ha sugerido que, en las sociedades con alto crecimiento económico, existe un cambio en los valores de los jóvenes, estos pasan de reivindicar valores modernos de supervivencia, relacionados con el trabajo y la seguridad, a 
reivindicar valores posmodernos de autoexpresión, relacionados con tolerancia, diversidad y apertura a nuevas experiencias (Inglehart, 2000). Por lo tanto, si los jóvenes no encuentran en el sistema político aquellos valores posmodernos, tenderían a alejarse de él (González et al., 2005). De allí la relevancia de estudiar las representaciones con respecto a elementos tan centrales como la vida política de los ciudadanos.

En relación con las investigaciones acerca de estas representaciones sociales, diversos estudios en contextos hispanoparlantes (Muñoz, Martínez y Muñoz, 2016; Rodríguez, Millán, Olvera, Moreno y González, 2004; Torres y Zubieta, 2015) tienden a mostrar que los conceptos políticos suelen tener un componente ideal al que se le adjudican una serie de valores, tales como la libertad o la igualdad, que se contrapone a un componente práctico negativo, en el que aparecen conceptos como mentiras y corrupción. De manera muy interesante, algunos estudios (Bruno, Barreiro y Kriger, 2011) sugieren que, en el imaginario colectivo, los conceptos de ciudadanía y política se han tornado en antagónicos. El primero relacionado con valores ideales positivos y el segundo con valores aplicados negativos.

En el contexto descrito, la educación juega un rol fundamental en preparar a las generaciones para enfrentar los desafíos que se erigen (Delors, 1996; PNUD, 2014a). Ello porque "no nacemos demócratas, aprendemos a serlo" (Cox y Castillo, 2015, p. 7); y se requiere de un ámbito común que trascienda los espacios en competencia y las particularidades de cada familia; "y la escuela es el espacio privilegiado de esa experiencia" (Peña, 2015, p. 35). Como espacio fundamental de socialización, la institución escolar es una experiencia permanente de aprendizaje ciudadano, pues contribuye a configurar "actitudes, emociones, valores, creencias, conocimientos y capacidades, tanto individuales como colectivas, que posibilitan las identidades y prácticas ciudadanas" (Ocampo-Talero, Méndez-París y Pavajeau-Delgado, 2008, p. 846). En tal sentido, la escuela puede constituirse como una entidad liberadora del individuo o reproductora de un orden social establecido, limitante de la ciudadanía, al menos de aquella que actualmente se piensa. De hecho, históricamente, ha predominado esto último (Apple, 1996, 1997; Freire, 1998; Giroux, 1993; Magendzo, 2004).

Para Marchesi (comunicación personal, junio de 2007), los estudios han diferenciado tres enfoques que asumen carácter complementario: la educación a través de la ciudadanía, para la ciudadanía y sobre la ciudadanía. Especialmente las dos últimas interpelan a la escuela y al rol que le cabe en dicho proceso. A su vez, eso remite, entre otros focos, a la formación inicial docente y la incorporación de la dimensión ciudadana como componente 
sustantivo de la formación de educadoras y educadores (Aranda, 2011; Cerda et al., 2004; Fernández-Herrería y López-López, 2014; Reyes et al., 2013;). Pese a ello, los estudios generalmente se han enfocado en la relación entre docentes y sus estudiantes, y rara vez indagan en las percepciones de los estudiantes de pedagogía, especialmente en Chile (e.g., Reyes et al., 2013; Treviño, Béjares, Villalobos y Naranjo, 2016). En un estudio reciente, también se ha recurrido a las percepciones de los estudiantes, consultando por las fuentes usadas para formar su opinión política actual (Muñoz, Martínez y Muñoz, 2016). Debe destacarse que, entre las fuentes reportadas, aparecen los medios de comunicación, la familia, y el debate social, pero no hay mención a la escuela ni al profesorado.

Dados estos antecedentes, esta investigación se ha propuesto indagar en las representaciones sociales de estudiantes de pedagogía de una universidad regional del sur de Chile, con miras a generar un insumo básico que nos permita evaluar propuestas de formación ciudadana para fortalecer los procesos de formación inicial docente.

\section{Metodología}

El estudio se fundó en un diseño transeccional descriptivo, con un enfoque metodológico cualitativo que utilizó el método de redes semánticas naturales (Valdés, 1998). Este permite el acceso empírico a la organización cognitiva del conocimiento, pues proporciona datos referidos a la organización e interpretación interna de un conjunto de significantes. Al dar cuenta de una estructura mental, la técnica de redes semánticas permite aprehender las representaciones sociales.

Se aplicó un muestreo intencionado a 249 estudiantes pertenecientes a 9 de las 10 carreras que constituyen la Facultad de Educación de una universidad regional del sur de Chile, durante las tres primeras semanas de diciembre de 2014. Excluyó a estudiantes de la carrera de Pedagogía en Educación Básica Intercultural Bilingüe, por la imposibilidad de conciliar horarios de aplicación, dado que estaban en proceso de evaluaciones finales.

El instrumento que se aplicó estuvo compuesto de dos secciones. La primera, denominada identificación, pesquisó información sociodemográfica e indagó en los contextos que, según los participantes, ejercieron mayor influencia en su aprendizaje sobre ciudadanía y democracia. La segunda sección expuso las palabras y frases estímulos: democracia, ciudadanía, democracia en Chile y Partidos políticos en Chile.

Para la segunda sección, la instrucción dada fue la siguiente: "Primero anote cinco palabras que relacione con las siguientes frases/conceptos. Luego, enumere las palabras en 
orden de importancia, del 1 al 5, tomando en cuenta que 1 es el más importante y 5 es el menos importante (escriba el número entre el paréntesis). No utilice más de una palabra en cada línea." A través de las dos primeras palabras (democracia y ciudadanía) se intentó indagar en las representaciones teóricas o ideales sobre dichos conceptos, por ello, se explicitó en las instrucciones escritas y orales que se dieron a los participantes. Con ello, se pretendió evitar que estos relacionaran los conceptos con alguna realidad determinada. En cambio, con los dos siguientes reactivos (democracia en Chile y Partidos políticos en Chile), se pretendió inquirir en las representaciones sociales referidas a las experiencias democráticas en las cuales viven o experimentan los participantes. Por ello, la presentación de dichas expresiones fue precedida por una instrucción que explicaba esto.

El análisis se estructuró fundamentándose en cuatro valores. El valor J, correspondiente al número total de definidores distintos en la red para cada uno de los grupos. El valor $\mathrm{M}$, correspondiente al peso semántico, obtenido como producto de la frecuencia de un nodo por su valor semántico (asignado en una escala del 1 a 5). El valor M da cuenta de la significatividad de los conceptos declarados. El grupo SAM presenta los 15 conceptos con mayor peso semántico para cada palabra o frase estímulo. Por último, el valor FMG representa la distancia semántica, expresada como la puntuación porcentual de las 15 palabras con mayor peso semántico (Valdés, 1998).

La muestra se compuso de estudiantes que llevaban entre 1 y 7 años en la universidad ( $\bar{X}=3,71$ años; $s=1,521)$, cuyas edades oscilaron entre los 18 y los 41 años, con un promedio de 22,7 años $(s=2,909)$, mayoritariamente de género femenino $(65,3 \%)$ y de origen urbano (85,5 \%). Al respecto, cabe señalar que, mientras un $34 \%$ provenía de Temuco y un 4,9\% provenía de Santiago, el 61,1\% restante declaró provenir de distintas comunas del país. En ese sentido, existe una alta heterogeneidad en la comuna de origen de la muestra. Los datos sociodemográficos se resumen en la Tabla 1.

Tabla 1

Caracterización sociodemográfica

\begin{tabular}{|c|c|c|c|c|}
\hline & Mínimo & Máximo & Media & $\begin{array}{c}\text { Desviación } \\
\text { estándar }\end{array}$ \\
\hline Edad en años & 18 & 41 & 22,75 & 2,909 \\
\hline Años en la universidad & 1 & 7 & 3,71 & 1,521 \\
\hline Años en Temuco & 0 & 38 & 10,02 & 9,618 \\
\hline & \multicolumn{2}{|c|}{ Masculino } & \multicolumn{2}{|c|}{ Femenino } \\
\hline Género & \multicolumn{2}{|c|}{$34,7 \%$} & \multicolumn{2}{|c|}{$65,3 \%$} \\
\hline & \multicolumn{2}{|c|}{ Urbano } & \multicolumn{2}{|c|}{ Rural } \\
\hline Origen & \multicolumn{2}{|c|}{$85,5 \%$} & \multicolumn{2}{|c|}{$14,5 \%$} \\
\hline
\end{tabular}

Elaboración propia de los autores (2016). 
Se estimó, además, el nivel socioeconómico usando el método Adimark/ESOMAR desarrollado en Chile por la consultora Adimark (2000), que se basa en la respuesta a dos preguntas (nivel de estudio y trabajo de la persona que aporta el mayor ingreso del hogar) para realizar una aproximación confiable y sencilla al estrato al que pertenece la familia de los encuestados. Los resultados indican que la muestra concentró estudiantes de clase media, con una leve tendencia hacia los estratos socioeconómicos más bajos (gráfico 1). Mientras solo un 4,3\% de la muestra pertenece a los estratos altos y muy altos, los estudiantes que provienen de familias ubicadas en el estrato bajo alcanza un 8,9\%. Esto es coherente con la tendencia de la universidad, que se caracteriza por tener un alumnado perteneciente a los tres quintiles más bajos.

\section{Gráfico 1.}

Distribución del NSE estimado

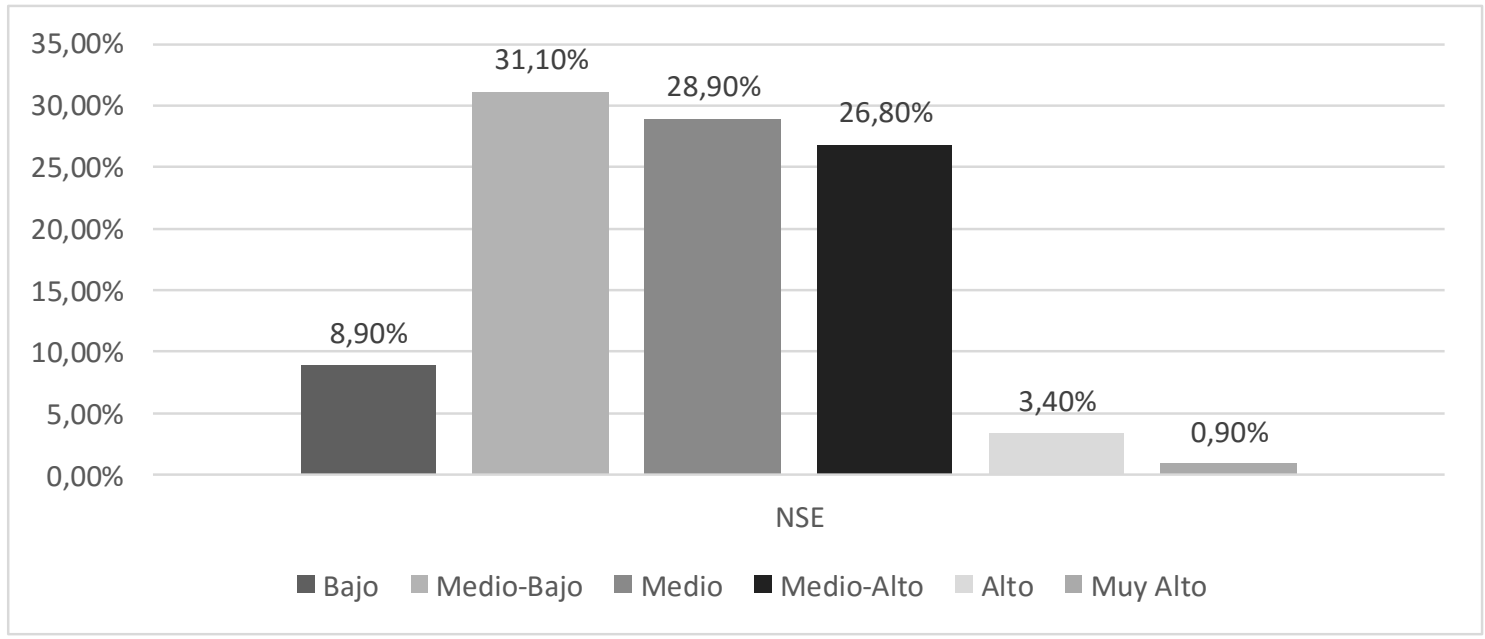

Elaboración propia de los autores (2016).

En un apartado especial, se les solicitó además a los participantes ordenar tres contextos cotidianos (familia, escuela, universidad) según su relevancia en su formación política y ciudadana. Como era esperable, el contexto que más veces aparece como muy relevante fue la familia (67,2\%); el menos relevante es, precisamente, la universidad $(48,6$ \%) (Ver gráfico 2). 
Gráfico 2.

Relevancia de contextos de aprendizaje en la formación ciudadana

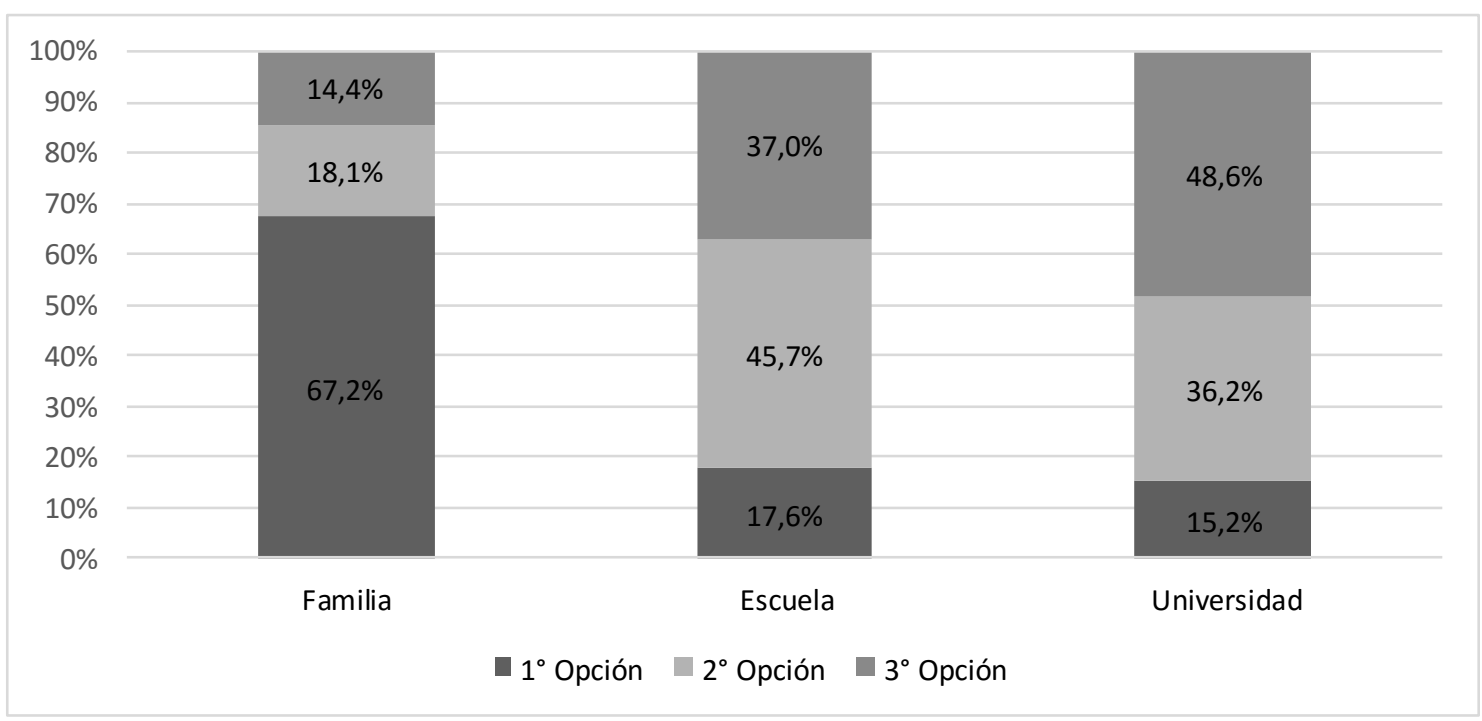

Elaboración propia de los autores (2016).

\section{Resultados y discusión}

Los resultados alcanzados evidencian, en primer lugar, una valoración positiva de la democracia por parte de los participantes (ver Tabla 2). Desde los conceptos empleados emergen representaciones que se condicen con el sentido de comunidad política, considerada como una base fundamental para la existencia de la democracia (Lizcano, 2012). También, términos como participación, opiniones, elecciones, pueblo y comunidad relevan, por una parte, el valor que tiene lo público como espacio de expresión de la democracia (Taguenca, 2012). Por otra, desde palabras como familia, libertad y expresión, se vislumbra el espacio privado como entidad desde la cual emerge la opinión de los ciudadanos que construyen espacios de diálogo (Habermas, 1998). Otros términos utilizados para representarla aluden a valores como la igualdad, la libertad, el respeto, la tolerancia y la justicia, los que, de acuerdo con la teoría, son rasgos definitorios de la democracia (Rawls, 1995a, 1995b; Walzer, 1996; MacIntyre, 2004). En estas representaciones, la palabra igualdad es el núcleo semántico, desplazando a un segundo lugar a la libertad como valor representativo de la democracia. No es posible indagar a través de la información recogida sobre los significados más profundos de dicho núcleo. Una opción es verla como igualdad ante la ley; otra, trascendiendo los marcos jurídico-legales asumiendo la connotación de igualdad social. Sería recomendable hacer más indagaciones en este punto. Sin embargo, es posible sostener que predomina una valoración donde destaca lo colectivo. Se mira la 
comunidad y se la representa asociada con la igualdad, aspectos que se condicen con lo sostenido por autores como Walzer (1996) y Maclntire (2004). La igualdad como valor se construye sobre la base de la mirada del todo, situando al individuo en relación con la comunidad; la libertad, en cambio, pone al individuo por delante (Rawls, 1995b). La igualdad también debe ponerse en contraste con su antónimo: desigualdad. Ello porque esta percepción negativa de la democracia chilena corresponde a participantes cuya caracterización socioeconómica los sitúa en el nivel socioeconómico medio bajo, rasgo relevado por otros estudios. (PNUD, 2014b; Redón, 2010; Taguenca y Lugo, 2011)

Asimismo, resulta interesante la asociación del concepto con las palabras pueblo, oportunidades, opiniones, expresión y participación. Ello remite a la existencia de un sistema de base popular que permite a las personas opinar y expresarse, acciones vinculadas a lo comunitario. Por último, las palabras Política, Gobierno, Derecho, Elecciones y Voto aluden a las expresiones político-jurídicas estructurales de la democracia y los mecanismos establecidos para orientar la participación de la gente. En tal sentido, estos últimos términos guardan más relación con las ideas liberales, que han sido instaladas en el país desde el retorno a la democracia. En resumen, mirada en una perspectiva ideal, los estudiantes representan a la democracia como un sistema apropiado para la organización de la vida social. Se trata, en esencia, de un sistema que asegura la posibilidad de movimiento y de actuación directa que parece acercarse más al enfoque comunitarista (nótese el carácter cohesionador de la palabra "pueblo"), pero inmerso en un sistema que vela por el cumplimiento de principios de justicia básicos muy similares a los propuestos por Rawls (1995b). Independientemente de un mayor predominio del matiz liberal o comunitario en algunos sujetos, es posible sostener que la democracia y la ciudadanía son legitimadas y validadas por todos como expresiones de proyecto social. 
Tabla 2.

Conjunto SAM de los conceptos democracia y ciudadanía

\begin{tabular}{|l|l|c|c|c|l|c|c|}
\hline \multicolumn{4}{|c|}{ DEMOCRACIA $(\mathrm{J}=215)$} & \multicolumn{4}{c|}{ CIUDADANIA $(\mathrm{J}=235)$} \\
\hline $\mathrm{N}$ & Palabra definitoria & $\mathrm{M}$ & $\mathrm{FGM}$ & $\mathrm{N}$ & Palabra definitoria & $\mathrm{M}$ & $\mathrm{FGM}$ \\
\hline 1 & Igualdad & 444 & $100,0 \%$ & 1 & Derecho & 457 & $100,0 \%$ \\
2 & Libertad & 404 & $91,0 \%$ & 2 & Deber & 320 & $70,0 \%$ \\
3 & Derecho & 281 & $63,3 \%$ & 3 & Personas & 191 & $41,8 \%$ \\
4 & Respeto & 217 & $48,9 \%$ & 4 & Respeto & 186 & $40,7 \%$ \\
5 & Participación & 189 & $42,6 \%$ & 5 & Participación & 172 & $37,6 \%$ \\
6 & Opiniones & 171 & $38,5 \%$ & 6 & Sociedad & 111 & $24,3 \%$ \\
7 & Elecciones & 112 & $25,2 \%$ & 7 & Responsabilidad & 95 & $20,8 \%$ \\
8 & Justicia & 96 & $21,6 \%$ & 8 & Comunidad & 91 & $19,9 \%$ \\
9 & Pueblo & 96 & $21,6 \%$ & 9 & Compromiso & 73 & $16,0 \%$ \\
10 & Voto & 66 & $14,9 \%$ & 10 & Igualdad & 73 & $16,0 \%$ \\
11 & Expresión & 60 & $13,5 \%$ & 11 & Unidad & 62 & $13,6 \%$ \\
12 & Política & 60 & $13,5 \%$ & 12 & Convivencia & 59 & $12,9 \%$ \\
13 & Tolerancia & 60 & $13,5 \%$ & 13 & Opiniones & 54 & $11,8 \%$ \\
14 & Oportunidades & 55 & $12,4 \%$ & 14 & Voto & 53 & $11,6 \%$ \\
15 & Gobierno & 52 & $11,7 \%$ & 15 & Familia & 52 & $11,4 \%$ \\
\hline
\end{tabular}

Elaboración propia de los autores (2016).

Esta idea se refuerza al analizar la forma en la que los estudiantes representan la palabra ciudadanía, cuyo núcleo figurativo es la palabra derecho; es decir, una facultad otorgada a quienes tienen el estatus de ciudadano. La palabra deber, que figura en segundo lugar de relevancia, alude a una obligación en el ejercicio de la ciudadanía. Este doble carácter con el que se representa el término permite sostener que los sujetos reconocen con claridad los alcances básicos de la ciudadanía en una democracia. El uso de palabras como personas, participación, responsabilidad, comunidad, compromiso y unidad sugieren significados en sintonía con ideas comunitaristas, tal como se proponía en el párrafo anterior. Esto porque dichos términos relevan el carácter colectivo de la ciudadanía como actividad ejercida con miras al bien de la comunidad, a la cual se subordina cada sujeto individual como un deber ineludible. Esto resulta consonante con algunas propuestas que sugieren que los jóvenes en general no muestran desinterés por la política, sino por las vías tradicionales de política formal (INJUV, 2013). No obstante, se ha sugerido que la juventud chilena tiene un creciente interés por la participación a nivel comunitario (Muñoz, Martínez y Muñoz, 2016), interés que puede relacionarse precisamente con un sentimiento de mayor eficiencia política, representatividad y transparencia. Asimismo, expresiones como respeto, opiniones y voto sugieren representaciones asociadas a visiones más liberales de la ciudadanía. Aquí, 
sus alcances son más restringidos y se asocian principalmente con la práctica del voto y la libertad para emitir opiniones en un marco de derecho, en sintonía con los rasgos expuestos por Bárcena (1997) cuando da cuenta de la doctrina liberal. Se aprecia aquí una fuerte asociación entre ambos conceptos estudiados, en los que se amalgama una mirada individualista-jurídica con una percepción comunitarista-social de la cualidad ciudadana. La palabra familia, que figura al final, sugiere la relación que esta institución social básica tiene como fundamento de la ciudadanía, como entidad socializadora de sus saberes y valores. Por último, más allá de las especificidades arriba expresadas, la ciudadanía es claramente representada por los sujetos como un fenómeno positivamente valorado para la vida social. Estas representaciones de la democracia y la ciudadanía quedan ligadas claramente a la ética (Cortina, 2011), y sintonizan con el tercer significado de la ciudadanía expuesto por Martins (2000) que alude al ciudadano situado, consciente y crítico frente a su entorno.

Además, y también como lo sugiere sistemáticamente la literatura, se evidencia un quiebre manifiesto entre la teoría y la práctica de la democracia en las representaciones que los participantes tienen de la organización política del país en que habitan (Bruno, Barreiro y Kriger, 2011). Es aquí donde democracia y ciudadanía vistas como proyecto social chocan con sus expresiones, en cuanto a realidades construidas históricamente (ver Tabla 3); ello porque proyecto y realidad no se condicen (Habermas, 1998). De hecho, el núcleo figurativo del reactivo que inquiere por la democracia en Chile está compuesto por la palabra desigualdad, la cual, además, se encuentra a gran distancia semántica de los demás conceptos. A este se añaden por lo menos otros 5 de los 15 términos del grupo SAM que tienen también claras denotaciones negativas: injusticia, corrupción, mentiras, inexistente y no representativa. A ellos habría que agregar que la palabra utopía, también presente en la red semántica, parece aludir a la democracia chilena como una pretensión irrealizable en comparación con la idealidad del concepto. Los otros términos asociados (derecho, libertad, respeto, participación, voto, deber, opiniones y elecciones) se condicen más con rasgos objetivos de la democracia chilena como estructura institucional. Es decir, no queda demostrada una valoración de la democracia chilena como un sistema promotor de vida buena, dispensador de dones para sus ciudadanos, idea que sí se expresa en la representación ideal del concepto. En ese sentido, aparece un reconocimiento a las cualidades institucionales de la democracia chilena, mientras queda al descubierto el descontento de los estudiantes con respecto a su funcionamiento político, que es visto como un sistema poco transparente que perpetúa las desigualdades socio-económicas del país 
(considérese las palabras "desigualdad", "injusticia", "corrupción", "mentiras" y "no representativa").

Aplicadas estas consideraciones al contexto estudiado, la base del análisis debe tomar en cuenta el devenir histórico en las últimas décadas. El retorno a la democracia en 1990 conllevó la reconstrucción del sistema político chileno. Sin embargo, la democracia mantuvo la institucionalidad política y la plataforma económica neoliberal heredada de un gobierno militar autoritario (Garretón, 2003; Salazar, y Pinto, 1999). Esto ha producido contradicciones y malestar en la ciudadanía y ha puesto en entredicho la legitimidad del orden democrático (Salazar y Pinto, 1999). Los términos usados por los sujetos del estudio revelan esta inconformidad con el sistema. Incluso, las palabras más descalificativas que se registraron bien pueden interpretarse como expresiones de desacato y la demanda de una legitimidad que solo se logra con mayor participación (Habermas, 1998; Salazar, 2009, 2012). Esta desigualdad que tanto mencionan los estudiantes puede explicarse desde la relación entre el liberalismo político y la dimensión económica que le sirve de base: el mercado, fundamentada por el liberalismo económico. Específicamente, el neoliberalismo. Las diferencias generadas desde el mercado no encuentran correlato en la supuesta igualdad esperada desde lo político. (Habermas, 1998)

Tabla 3.

Conjunto SAM de los conceptos democracia en Chile y partidos políticos en Chile

\begin{tabular}{|l|l|c|c|c|l|c|c|}
\hline \multicolumn{4}{|c|}{ DEMOCRACIA EN CHILE (J=412) } & \multicolumn{4}{|c|}{ PARTIDOS POLÍTICOS CHILE (J=375) } \\
\hline $\mathrm{N}$ & Palabra Definitoria & $\mathrm{M}$ & $\mathrm{FGM}$ & $\mathrm{N}$ & Palabra Definitoria & $\mathrm{M}$ & $\mathrm{FGM}$ \\
\hline 1 & Desigualdad & 285 & $100,0 \%$ & 1 & Corrupción & 189 & $100,0 \%$ \\
2 & Derecho & 95 & $33,3 \%$ & 2 & Mentiras & 157 & $83,1 \%$ \\
3 & Injusticia & 86 & $30,2 \%$ & 3 & Poder & 112 & $59,3 \%$ \\
4 & Libertad & 69 & $24,2 \%$ & 4 & Intereses & 98 & $51,9 \%$ \\
5 & Corrupción & 64 & $22,5 \%$ & 5 & Dinero & 80 & $42,3 \%$ \\
6 & Mentiras & 58 & $20,4 \%$ & 6 & Lucro & 72 & $38,1 \%$ \\
7 & Utopía & 51 & $17,9 \%$ & 7 & Desigualdad & 51 & $27,0 \%$ \\
8 & Respeto & 48 & $16,8 \%$ & 8 & No representativo & 49 & $25,9 \%$ \\
9 & Participación & 45 & $15,8 \%$ & 9 & Oportunismo & 48 & $25,4 \%$ \\
10 & Voto & 43 & $15,1 \%$ & 10 & Idealismo & 47 & $24,9 \%$ \\
11 & Inexistente & 41 & $14,4 \%$ & 11 & Ladrones & 41 & $21,7 \%$ \\
12 & Deber & 40 & $14,0 \%$ & 12 & Representatividad & 38 & $20,1 \%$ \\
13 & Opiniones & 40 & $14,0 \%$ & 13 & Falsos & 36 & $19,0 \%$ \\
14 & No representativa & 36 & $12,6 \%$ & 14 & Falsedad & 35 & $18,5 \%$ \\
15 & Elecciones & 33 & $11,6 \%$ & 15 & Inconsecuencia & 35 & $18,5 \%$ \\
\hline
\end{tabular}

Elaboración propia de los autores (2016). 
La expresión estímulo partidos políticos en Chile corrobora todavía con mayor claridad la tensión entre la democracia como idea con su expresión real de experiencia vivida. El núcleo de la red semántica corresponde a la palabra corrupción. La primera representación que emerge alude a este vicio común el cual, en mayor o menor medida, aqueja a las instituciones públicas, que es el aprovechamiento de las atribuciones y funciones para obtener un provecho particular. Esta impresión de descomposición de los partidos políticos se ve reforzada con el uso de otros términos tales como poder, intereses, oportunismo, lucro y dinero. Dichas representaciones coinciden con lo relevado por otros estudios (Rodríguez et al., 2004; Torres y Zubieta, 2015). Una segunda idea que se distingue es la falta de representación de los partidos políticos, aspecto que también aparece relevado en otros estudios que apuntan a una falta de sintonía de estas agrupaciones con la ciudadanía (e.g., Flores-González y García-González, 2014). Si se asume que los partidos políticos constituyen una base importante para consolidar la democracia, resulta preocupante la desaprobación que muestran los jóvenes por aquellos que operan en el país. La mayoría de los conceptos que componen el conjunto SAM (11 de 15) se vinculan con denotaciones claramente negativas. Inclusive, algunas expresiones, como ladrones y falsos, incurren en una abierta reprobación. En otras palabras, es posible sostener que el ámbito estudiado representa la idea de coexistencia tensa entre estabilidad superficial -dada por el orden político- en medio de una inestabilidad profunda, manifestada por la representación que tienen los ciudadanos de dicho orden (Salazar y Pinto, 1999). La respuesta en este tipo de contextos, según Rosanvallon (2007), es la gestación de mecanismos contrademocráticos destinados al ejercicio indirecto de la soberanía. También, la generación de luchas contrahegemónicas, asociadas a procesos de formación de identidad o bien, a demandas más funcionales que se libran en los espacios públicos y que desafían las leyes vigentes (Delgado, 2011; Janoschka, 2011; Sequera y Janoschka, 2012). El caso chileno presenta tendencias de este tipo en que algunos actores asumen la movilización social en detrimento de la participación electoral. De hecho, en la última década, el movimiento estudiantil producido en el espacio objeto de esta investigación es una clara evidencia de estos movimientos de rebeldía. Ciertamente, existen múltiples aproximaciones metodológicas que permiten abordar este fenómeno, pero un primer paso esencial consiste precisamente en indagar las formas que adoptan los conceptos políticos en las representaciones de los jóvenes. En este estudio, se ha optado por estudiar las representaciones sociales, entendidas como un mecanismo de orientación y apropiación del entorno social (Moscovici, 1979), enfocándonos principalmente en lo que se ha denominado el campo de 
representación, que organiza semánticamente la estructura de nuestras representaciones (Condor y Antaki, 2000), de modo que se pueda entender -y eventualmente intervenir- la forma en la que las representaciones modulan la construcción del conocimiento social (Castorina, 2008) en los futuros formadores del país. Si bien no es la pretensión de este estudio, cuyo carácter exploratorio limita su campo de acción, diseñar este tipo de intervenciones, es de destacar que los resultados que acaban de discutirse se alinean perfectamente con otros estudios similares realizados en Chile (Cárdenas et al., 2007) y en otros países hispanoparlantes, como Argentina (Bruno et al., 2011; Torres y Zubieta, 2015) y México (Rodríguez et al., 2004); es decir, una mirada polarizada de lo político, en la que se contrapone lo ideal con lo aplicado. En este caso, al forzar la mirada ideal del concepto de "democracia", se encuentra con que está generalmente asociado a valores positivos, muy en sintonía con el concepto idealizado de "ciudadanía"; sin embargo, al pedir que se evalúe la aplicación práctica en el caso chileno de democracia, esta valoración positiva se desvanece. Esto parece sugerir que no es el sistema político en sí mismo lo que genera un alejamiento de los jóvenes con respecto a la acción política tradicional (por ejemplo, ejercer el derecho a voto), sino un desencanto que se relaciona con una necesidad de mayor transparencia, participación directa y representación de la política partidista. La ciudadanía no diferencia a los políticos por su posicionamiento político en el eje izquierda-derecha porque ellos mismos no se encuadran con claridad en estos ejes, posiblemente a causa del desprestigio generalizado de los partidos políticos, desprestigio cuya raíz profunda tiene su origen en la misma recuperación de la democracia, y en la construcción de esta nueva esfera de poder, que se legitima de forma ficticia en procesos electorales, para abandonar luego cualquier espacio participativo de la ciudadanía (Flores-González y García-González, 2014). En una propuesta muy interesante, González y colaboradores (2005) han sugerido que los jóvenes en Chile han renunciado lentamente a los referentes políticos, alejándose de las categorías sociopolíticas más específicas representadas por los partidos, que satisfacen necesidades de inclusión y de diferenciación grupal, para identificarse en categorías más amplias de coaliciones políticas, que les permiten mantener una sensación de pertenencia grupal, a costa de su distintividad política.

Estas expresiones no solo demandan cambios al sistema político, sino, también, vías formativas que permitan canalizar este espíritu crítico por vías transformadoras, pero constructivas. La realidad de la época demanda miradas más amplias de la ciudadanía (Cortina, 1998; Garretón, 2003, Re, 2014), especialmente, y se valida la postura de verla como una categoría en constante actualización (Sequera y Janoschka, 2012); y ello reclama 
una acción fundamental desde el sistema educativo (Cox y Castillo, 2015). Si la escuela es el primer espacio de convergencia de distintas visiones de sociedad e interacción entre diversas concepciones de libertad y autoridad (Peña, 2015), cabe preguntarse por su impacto formativo. ¿Cómo fueron las experiencias ciudadanas vividas en la escuela por los sujetos estudiados? ¿Qué tan relevante fueron en relación con otras instancias de aprendizaje ciudadano vividas más allá de la escuela? ¿Ha sido la institución universitaria un eje relevante de su formación ciudadana? ¿Cómo integrar las ideas socio-políticas de estos futuros maestros y maestras de manera consistente en los procesos de formación ciudadana? Son interrogantes que emergen a la luz de esta investigación. Las representaciones pesquisadas constituyen una señal de alerta que obligan a repensar el rol que tradicionalmente ha jugado la escuela (Apple, 1996, 1997; Freire, 1998; Giroux, 1993; Magendzo, 2004). El cuestionamiento se extiende hacia la propia universidad, pues los movimientos estudiantiles han buscado vías alternativas de expresión, apelando a medidas de fuerza como paros y tomas.

La estructura educativa se ve así interpelada; y, por lo tanto, la institución universitaria. Particularmente, se erige como una instancia relevante de formación ciudadana, en especial, lo que la literatura denomina "educación a través de la ciudadanía". A diferencia de la escuela, quienes interactúan en el espacio universitario son ciudadanos de pleno derecho; la búsqueda de conocimiento, la deliberación y el diálogo son aspectos transversales de la idiosincracia universitaria -o debieran serlo.

Aunque no es el objetivo de este estudio establecer una relación entre formación ciudadana y formación universitaria, ciertamente, sí se busca posicionar la idea de que existe una necesidad que la universidad tome parte en este proceso. Hay estudios que avalan la necesidad de potenciar la formación inicial docente en términos de educación ciudadana (Reyes et al., 2013); esto tanto en términos institucionales como personales. En ese sentido, el trabajo de Cárcamo (2008) representa un avance importante. Reconocer que la labor académica y la trayectoria universitaria en todas sus aristas necesariamente requieren de un trato democrático dentro y fuera del aula de clases, que permita modular y modelar la conducta de todos sus actores, especialmente de sus estudiantes. Así, la vivencia de la democracia en un entorno realmente democrático permitiría a sus actores internalizar y asumir sus valores asociados, con altos beneficios en términos de calidad de vida de todos los actores involucrados (Lawrence, Ott y Bell, 2011; Macfarlane, 2007) y de fortalecimiento de la universidad como centro de formación integral. En ese sentido, esta investigación es un llamado de atención ante este fenómeno. 


\section{Conclusiones}

La presente investigación se fijó como objetivo describir las representaciones de democracia y ciudadanía de un grupo de estudiantes universitarios de pedagogía pertenecientes a una universidad chilena de carácter regional. Se indagó en los significados de democracia y ciudadanía, como conceptos ideales; también, en la democracia como expresión socio-histórica concreta. En este último caso, se buscó conocer las representaciones de la democracia chilena y los partidos políticos chilenos.

Al tenor de los resultados, una primera conclusión es la impresión positiva que los participantes manifiestan de la democracia y la ciudadanía como conceptos ideales. En ambos casos, las representaciones que emergieron se asocian con denotaciones positivas y necesarias para la adecuada organización de la sociedad. Se perciben algunos matices que sugieren en unos casos una mayor sintonía con ideas liberales, en otros, con visiones más cercanas al comunitarismo.

En segundo lugar, se constata la existencia de representaciones que dan cuenta de la existencia de un malestar con la democracia y los partidos políticos en Chile. La falta de representatividad, la desigualdad y la injusticia emergen como rasgos asociados al sistema democrático chileno. Destaca la ausencia de los rasgos positivos que aparecen asociados a la democracia como concepto ideal. En el caso de los partidos políticos, las representaciones los asocian con la corrupción, la defensa de intereses particulares y la desconexión con la ciudadanía.

De esto se desprende una clara implicancia social y política, relacionada con un quiebre importante entre las dimensiones de lo ideal y lo real, que se han cristalizado en una serie de movilizaciones sociales en Chile, especialmente durante la última década. Este malestar observado en los estudiantes de pedagogía del presente estudio es el malestar de una generación que busca acercar estas dos dimensiones. No es casualidad que uno de los movimientos sociales más fuertes y estables del país sea precisamente uno estudiantil, el cual, con altos y bajos, ha estado presente en la agenda política y mediática por lo menos desde el 2006, llevando incluso a algunas de sus figuras más emblemáticas a ganar elecciones parlamentarias.

El caso chileno es particularmente interesante, por cuanto las políticas liberales con las que se ha conducido el país, incluso desde el retorno a la democracia y con prevalencia de presidentes de izquierda, han conducido a una despolarización de la política en el sentido tradicional. Las representaciones que emergieron dan cuenta de nuevas ideas y actitudes. 
Considerarlas en pos de transformaciones que fortalezcan la democracia resulta ser una necesidad imperativa. Interpela a la sociedad toda y, especialmente, a su sistema educativo. En esa perspectiva, la institución universitaria no puede quedar al margen de dicha responsabilidad. Sin embargo, en el presente estudio, la universidad no parece jugar un rol importante en la formación ciudadana. Casi la mitad de los participantes consideraron la casa de enseñanza como el espacio menos relevante en ese sentido, algo que parece un contrasentido cuando se espera de ellos que formen ciudadanos responsables. Estudiar las representaciones de la ciudadanía con respecto a conceptos políticos básicos permite una mejor aproximación desde las instituciones de educación superior, y muy especialmente desde las formaciones iniciales docentes, para abordar el complejo tema de la formación ciudadana desde uno de sus pilares fundamentales: la universidad.

Un punto relevante por tener en cuenta es que los estudiantes que llegan a ella son ya ciudadanos por derecho propio y no ciudadanos en formación. La familia y la escuela debiesen ser espacios para integrar los primeros conocimientos y aplicaciones del funcionamiento político. Es bastante llamativo que más de un tercio de los participantes consideraron que la escuela fue la institución que menos les enseñó sobre democracia, lo que puede indicar un cierto fracaso de las políticas educacionales al respecto; ciertamente, los resultados en evaluaciones internacionales así lo señalan. En un país con altos niveles de machismo, una historia reciente marcada por el quiebre y la recuperación de la democracia, y alta desigualdad económica, la escuela debiese configurarse en un elemento fundamental para la recuperación de un clima democrático. En ese contexto, la universidad, especialmente las carreras de pedagogía, no puede quedar al margen de este proceso, pero cabe preguntarse cómo entregar herramientas para el funcionamiento político a individuos politizados. Frente a esta necesidad, se posiciona el desafío de distinguir y articular aquellos factores que resultan ser intervinientes en un proceso formativo de esta envergadura; una primera dimensión relevante es el vínculo familia y universidad. Difícil es concebir la forma en cómo tales entidades puedan entablar una relación de diálogo y aprendizaje mutuo en materia de colaboración para contribuir al desarrollo de una colectividad más comprometida con los asuntos de todos. No obstante, se piensa que debe desarrollarse una indagación en este ámbito. En el plano del espacio propiamente universitario, más que pensar en la gestación de modelos educativos desde una racionalidad meramente instrumental, resulta indispensable propiciar la profundización de la democratización de la universidad como espacio colectivo de participación y desarrollo. 
El presente estudio presenta una serie de limitaciones. Por una parte, la muestra se redujo a los estudiantes de Pedagogía de una universidad regional pública no estatal. Es posible que universidades con otros perfiles de estudiantes muestren otros resultados, por lo que sería conveniente replicar esta investigación en otras casas de estudio. Por otra parte, uno de los motivos por lo que resulta interesante un estudio de este tipo en el contexto geopolítico de la región de la Araucanía, es la diversidad étnica presente, con un fuerte componente indígena. Muy especialmente, debido a la tensión política que se ha mantenido relativamente constante a través del tiempo, con fuertes movimientos reivindicadores del pueblo mapuche. No obstante, esta investigación, por su alcance exploratorio y por su extensión actual, no pudo abordar esta variable. Sería de sumo interés incluirla en futuros estudios, comparando las representaciones sociales de conceptos políticos básicos en población mapuche y no mapuche.

La reflexión con respecto a la pertinencia de integrar la formación ciudadana en la universidad es un debate que trasciende su función meramente formativa o investigativa. Es un debate ético-moral según el impacto y la responsabilidad social de estas instituciones de cara a las crecientes demandas y necesidades del país. Un debate que, sin duda, debe ser abordado con responsabilidad ante la evidencia clara de que Chile -al igual que otros países de la región- no ha logrado recomponerse de la fuerte herencia militar que ha dejado la dictadura. No se puede dejar de insistir en ello: para recuperar realmente la democracia, no basta con cambiar de un régimen autoritario a uno parlamentario. Lo que se requiere es un cambio cultural, un aspecto en el que el sistema de educación formal -especialmente la universidad, que forma a los futuros maestros y maestras del país- es clave; y está en deuda con la sociedad.

\section{Referencias}

Adaros, Abner, Manzano, Rubén, Mella, Saúl y Montero, Daniela. (2010). Juventud y bicentenario. Estudiantes secundarios, procesos de construcción de identidad ciudadana y discursos marginales. La Serena-Coquimbo, 2006-2009. Ultima Década, 18(33), 139-165.

Adimark. (2000). El Nivel Socio Económico Esomar Manual de Aplicación. Recuperado de http://www.microweb.cl/idm/documentos/ESOMAR.pdf

Agüero, Javier, Araya, Iliana, Marín, Juan, Molina, Silvia y Rojas, Francisco. (2011). La Enseñanza de los Estudios Sociales y la Educación Cívica: hacia una profesión interdisciplinar. Diálogos, Revista Electrónica de Historia, 12(2), 26-48. 
Aparecido, Ednaldo y Borba, Julián. (2011). Participación y democracia en América Latina: los determinantes individuales de la participación política. Foro Internacional, 51(2), 242-270.

Apple, Michael. (1996). El conocimiento oficial. La educación democrática en una era conservadora. Buenos Aires: Paidós.

Apple, Michael. (1997). Educación y poder. Barcelona: Paidós.

Aranda, Virginia. (2011). Reflexión y análisis de políticas y prácticas innovadoras a la luz de las representaciones sociales y de la necesidad de una educación intercultural en la formación inicial docente. Estudios Pedagógicos, 37(2), 301-314.

Aravena, Pedro. (2011). ¿Es el crecimiento económico suficiente para apoyar la democracia? Lecciones del caso chileno. Estudios Internacionales, 44(170), 7-30.

Bárcena, Fernando. (1997). El oficio de la ciudadanía. Introducción a la educación política. Barcelona: Paidós.

Berger, Peter y Luckmann, Thomas. (1991). The social constuction of reality. A treatise in the sociology of knowledge. Londres: Penguin Books.

Bernardo, Marcia. (2014). Produtivismo e precariedade subjetiva na universidade pública: o desgaste mental dos docentes. Psicologia \& Sociedade, 26(especial), 129-139.

Bruno, Daniela, Barreiro, Alicia y Kriger, Miriam. (2011). Representaciones sociales de la política en los jóvenes : Corrupción institucional y mentira. Kairos, 15(28), 1-16.

Cabalin, Cristian. (2012). Identidad cultural y ciudadanía en los sectores pobres de Santiago de Chile. Perfiles Latinoamericanos, 20(40), 123-142.

Candia, Lilian y Gomes, Paulo. (2013). Ciudadanía, educación y la realidad brasileña: Puntos de debate y encaminamientos. Estudios Pedagógicos, 39(2), 41-54.

Cárcamo, Héctor y Méndez, Pablo. (2016). Imaginarios sociales en torno al comportamiento ciudadano docente: una mirada desde la formación inicial docente. Actualidades $\begin{array}{lllll}\text { Investigativas en } & \text { Educación, } & \text { 16(1), } & \text { DOI: }\end{array}$ http://dx.doi.org/10.15517/aie.v16i1.21969

Cárcamo, Héctor. (2008). Importancia atribuida al desarrollo de la ciudadania en la formacion inicial docente. Estudios Pedagógicos, 34(2), 29-43.

Cárdenas, Manuel, Parra, Luis, Picón, Juan, Pineda, Héctor y Rojas, Rodrigo. (2007). Las Representaciones Sociales de la Política y la Democracia. Ultima Década, (26), 53-78.

Carlin, Ryan. (2011). Distrusting Democrats and Political Participation in New Democracies: Lessons from Chile. Political Research Quarterly, 64(3), 668-687. 
Castorina, José. (2008). El impacto de las representaciones sociales en la psicología de los conocimientos sociales: problemas y perspectivas. Cadernos Da Pesquisa, 38(135), 757-776.

Centeno, Gabriel. (2008). Globalización, educación y competencias. Ábaco, (55/56), 53-67.

Cerda, Ana, Egaña, Loreto, Magendzo, Abraham, Santa Cruz, Eduardo y Varas, René. (2004). El complejo camino de la formación ciudadana. Una mirada a las prácticas docentes. Santiago: LOM Ediciones - PIIE.

Comisión Formación Ciudadana, Chile. (2004). Informe final. Santiago: Ministerio de Educación de Chile.

Condor, Susan y Antaki, Charles. (2000). Cognición social y discurso. En Teun Van Dijk (Ed.), El discurso como estructura y proceso (pp. 453-489). Barcelona: Gedisa.

Congreso Nacional de Chile. (2016). Ley Núm. 20.911. Crea el Plan de Formación Ciudadana para los Establecimientos Educacionales reconocidos por el Estado. Santiago: Diario Oficial de la República de Chile.

Cortina, Adela. (1998). Ciudadanos del mundo. Hacia una teoría de la ciudadanía. Madrid: Alianza Editorial.

Cortina, Adela. (2011). Ciudadanía democrática: ética, política y religión. XIX Conferencias Aranguren. Isegoría, (44), 13-55.

Cox, Cristian y Castillo, Juan. (2015). Aprendizaje de la ciudadanía. Contextos, experiencias y resultados. Santiago: Ediciones Universidad Católica de Chile.

De la Cuadra, Fernando. (2013). Apontamentos sobre o conflito e os movimentos sociais no Chile. Dilemas: Revista de Estudos de Conflito E Controle Social, 6(4), 581-602.

Delgado, Ricardo. (2011). Educación para la ciudadanía desde la acción colectiva. Magis, Revista Internacional de Investigación En Educación, 4(7), 201-210.

Delors, Jacques. (1996). La educación encierra un tesoro. México: Unesco.

Díaz, Francisco y Sierra, Lucas. (2012). Democracia con partidos. Informe para la reforma de los partidos políticos en Chile. Santiago: CEP - Cieplan.

Domingo, Pilar. (2009). Ciudadanía , derechos y justicia Latina en América. Ciudadanizaciónjudicialización de la política. Revista CIDOB D’ Afers Internacionals, (85/86), 33-52.

Dorantes, Gerardo. (2014). Procesos electorales y democracia: la construcción de una agenda de campaña política. Convergencia. Revista de Ciencias Sociales, 21(64), 143-168.

Errázuriz, Luis. (2009). Dictadura militar en Chile. Antecedentes del golpe estético-cultural. Latin American Research Review, 44(2), 136-157. 
Fernández-Herrería, Alfonso y López-López, María del Carmen. (2014). Educar para la paz. Necesidad de un cambio epistemológico. Convergencia. Revista de Ciencias Sociales, 21(64), 117-142.

Flores-González, Luis y García-González, Carolina. (2014). Paradojas de la participación juvenil y desafíos de la educación ciudadana en Chile. Magis, Revista Internacional de Investigación En Educación, 6(13), 31-48.

Freire, Paulo. (1998). Pedagogía de la esperanza. México: Siglo Veintiuno.

Garretón, Manuel. (2003). Incomplete Democracy. Polítical Democratization in Chile and Latin America. Chapel Hill: University of North Carolina Press.

Giroux, Henry. (1993). La escuela y la lucha por la ciudadanía. México: Siglo Veintiuno.

González, Teresa. (2014). La educación cívica en España: Retrospectiva y perspectiva. Historia Da Educacao, 18(42), 115-130.

González, Roberto, Manzi, Jorge, Cortés, Flavio, Torres, David, De Tezanos, Pablo, Aldunate, Nerea, Aravena, María y Sáiz, José Luis. (2005). Identidad y actitudes políticas en jóvenes universitarios: El desencanto de los que no se identifican políticamente. Revista de Ciencia Política, 25(2), 65-90.

Gozálvez, Vicent. (2011). Educación para la ciudadanía democrática en la cultura digital. Comunicar, 18(36), 131-138.

Guichot, Virginia. (2013). Participación, ciudadanía activa y educación. Teoría de La Educación. Revista Interuniversitaria, 25(2), 25-47.

Habermas, Jurgen. (1975). Problemas de legitimación en el capitalismo tardío. Buenos Aires: Amorrortu Editores.

Habermas, Jurgen. (1998). Facticidad y validez. Sobre el derecho y el Estado democrático de derecho en términos de teoría del discurso. Madrid: Trotta.

Hewstone, Miles. (1982). Intergroup relations and attribution processes. En Henri Tajfel (Ed.), Social identity and intergroup behavior (pp. 99-133). Cambridge: Cambridge University Press.

Hewstone, Miles. (1988). Attributional bases of intergroup conflict. En Wolfgang Stroebe, Arie Kruglansky, Daniel Bar-Tal, y Miles Hewstone (Eds.), The social psychology of intergroup conflict: Theory, research and applications (pp. 47-71). Berlín: SpringerVerlat.

Ibáñez, Tomás. (1988). Ideologías de la vida cotidiana. Barcelona: Sendai.

Inglehart, Ronald. (2000). Globalization and postmodern values. The Washington Quarterly, 23(1), 215-228. 
Instituto Nacional de La Juventud - INJUV, Chile. (2013). Séptima encuesta nacional de juventud 2012. Recuperado de http://www.injuv.gob.cl/portal/wpcontent/files_mf/septimaencuestanacionaljuventud2.pdf

Janoschka, Michael. (2011), Geografías urbanas en la era del neoliberalismo. Una conceptualización de la resistencia local a través de la participación y la ciudadanía urbana. Investigaciones Geográficas (Mx), (76), 118-132.

Jara, Camila. (2014). Democratic Legitimacy under Strain? Declining Political Support and Mass Demonstrations in Chile. European Review of Latin American and Caribbean Studies, (97), 25-50.

Larraín, Jorge. (2011). ¿América Latina moderna? Globalización e identidad. Santiago: LOM.

Lawrence, Janet, Ott, Molly y Bell, Alli. (2011). Faculty organizational commitment and citizenship. Research in Higher Education, 53(3), 325-352.

Lizcano, Franscisco. (2012). Democracia directa y democracia representativa. Convergencia. Revista de Ciencias Sociales, (60),145-175.

Macfarlane, Bruce. (2007). Defining and rewarding academic citizenship: the implications for university promotions policy. Journal of Higher Education Policy and Management. 29(3), 261-273.

Maclntyre, Alasdair. (2004). Tras la virtud. Barcelona: Crítica.

Magendzo, Abraham. (2004). Formación ciudadana. Bogotá: Cooperativa Editorial Magisterio.

Martínez, Miquel. (2011). Educación, valores y democracia. Revista de Educación, (número especial), 15-19.

Martins, Marcos. (2000). Uma "catarsis" no conceito de cidadania: do cidadáo cliente à cidadania com valor ético-político. Revista de Ética, 2(2), 106-118.

Moscovici, Serge. (1979). El psicoanálisis, su imagen y su público. Buenos Aires: Editorial Huemul, S.A.

Moscovici, Serge. (2001). Social representations: Essays in social psychology. Nueva York: New York University Press.

Muñoz, Carlos, Martínez, Rosendo y Muñoz, Carlos. (2016). Percepciones del estudiantado sobre la política, los partidos políticos y las personas dedicadas a la política al finalizar la educación secundaria en Chile. Revista Electrónica EDUCARE, 20(1), 1-16. doi: 10.15359/ree.20-1.17.

Ocampo-Talero, Angélica, Méndez-París, Sara y Pavajeau-Delgado, Carol. (2008). Las subjetividades como centro de la formación ciudadana. Revista Universitas Psychologica. 7(3), 837-851. 
Passarelli, Gianluca. (2011). Presidential and Congressional Elections in Chile, December 2009 and January 2010. European Review of Latin American and Caribbean Studies. 91, 71-80.

Peña, Carlos. (2015). Escuela y vida cívica. En Cristian Cox y Juan Castillo (Eds.), Aprendizaje de la ciudadanía. Contextos, experiencias y resultados (pp. 25-50). Santiago: Ediciones Universidad Católica de Chile.

Programa de las Naciones Unidas para el Desarrollo - PNUD. (2002). Informe sobre Desarrollo Humano 2002. Madrid: Mundi-Prensa.

Programa de las Naciones Unidas para el Desarrollo - PNUD. (2004). La democracia en América Latina: Hacia una democracia de ciudadanas y ciudadanos. (2a ed.). Buenos Aires: Aguilar, Altea, Taurus, Alfaguara.

Programa de las Naciones Unidas para el Desarrollo. (2011). El estado de ciudadanía. Transformaciones, logros y desafíos del Estado en América Latina en el siglo XXI. Buenos Aires: Sudamericana.

Programa de las Naciones Unidas para el Desarrollo. (2014a). Ciudadanía política. Voz y participación ciudadana en América Latina. Buenos Aires: Siglo Veintiuno.

Programa de las Naciones Unidas para el Desarrollo. (2014b). Resumen Informe sobre Desarrollo Humano 2014. Sostener el Progreso Humano: reducir vulnerabilidades y construir resiliencia. Washington DC: Programa de las Naciones Unidas para el Desarrollo.

Puig, Josep, Gijón, Mónica, Martín, Xus y Rubio, Laura. (2010). Aprendizaje-servicio y educación para la ciudadanía. Revista de Educación, (número extraordinario), 45-67.

Rawls, John. (1995a). Liberalismo político. México: Fondo de Cultura Económica.

Rawls, John. (1995b). Teoría de la justicia. México: Fondo de Cultura Económica.

Re, Arianna. (2014). Globalización y ciudadanía en América Latina. Quaderns-E. 19(1), 220234.

Redón, Silvia. (2010). La escuela como espacio de ciudadanía. Estudios Pedagógicos, 36(2), 213-239.

Reyes, Leonora, Campos, Javier, Osandón, Luis y Muñoz, Carlos. (2013). El profesorado y su rol en la formación de los nuevos ciudadanos: desfases entre las comprensiones , las actuaciones y las expectativas. Estudios Pedagógicos, 39(1), 217-237.

Rodríguez, Óscar, Millán, Angélica, Olvera, Lucía, Moreno, Berenice y González, Sandra. (2004). Representación social de la democracia: las prácticas invisibles. Polis, 1(4), 125-142.

Rosanvallon, Pierre. (2007), La contrademocracia. La política en la era de la desconfianza. Buenos Aires: Manantial. 
Salazar, Gabriel. (2009). Del poder constituyente de asalariados e intelectuales (Chile, siglos $X X$ y $X X I)$. Santiago: LOM.

Salazar, Gabriel. (2012). Movimientos sociales en Chile: Trayectoria histórica y proyección política. Santiago: Uqbar editores.

Salazar, Gabriel y Pinto, Julio. (1999). Historia contemporánea de Chile I. Estado, legitimidad, ciudadanía. Santiago: LOM.

Sequera, Jorge y Janoschka, Michael. (2012). Ciudadanía y espacio público en la era de la globalización neoliberal. Arbor, 188(755), 515-527.

Taguenca, Juan. (2012), La opinión política de los jóvenes universitarios de Hidalgo, México. Un análisis desde la teoría de campo. Convergencia. Revista de Ciencias Sociales, (60),45-77.

Taguenca, Juan y Lugo, Bernabé. (2011). Percepción de la democracia de los jóvenes mexicanos. Política y Gobierno, 18(2), 179-230.

Torres, Cynthia y Zubieta, Elena. (2015). Consenso y divergencias en las representaciones sociales de la dirigencia política. Revista de Psicología, 33(1), 87-128.

Treviño, Ernesto, Béjares, Consuelo, Villalobos, Cristóbal y Naranjo, Eloísa. (2016). Influence of teachers and schools on students' civic outcomes in Latin America. The Journal of Educational Research, 1-15. doi: 10.1080/00220671.2016.1164114.

Valdés, José. (1998). Las Redes Semánticas Naturales: Usos y Aplicaciones en Psicología social. México: Universidad Nacional Autónoma de México.

Walzer, Michael. (1996). Moralidad en el ámbito local e internacional. Madrid: Alianza Editorial. 